\title{
Nonlinear waves in an elastic guide interacting with an elastic-inertial foundation
}

\author{
Vladimir Erofeev ${ }^{1, *}$, Daniil Kolesov ${ }^{1}$, and Anna Leonteva $^{1}$ \\ ${ }^{1}$ Mechanical Engineering Research Institute of RAS, Nizhny Novgorod, Russia
}

\begin{abstract}
This paper deals with the study of the transverse wave propagation in a string resting on an elastic-inertial foundation. A selfconsistent dynamic problem is herein considered for a system composed of a one-dimensional flexible guide (string) and an elastic-inertial foundation. As a foundation model the Vesnitsky's model was chosen. The set of equations is reduced to one fourth-order cubic-nonlinear equation relative to transverse displacements of the string. Depending on the string and the elastic-inertial foundation mass ratio, the evolutionary equation has the following three extreme events: the modified Ostrovsky equation, the Riemann equation with a cubic nonlinearity, the equation for an anharmonic oscillator with a cubic nonlinearity.
\end{abstract}

Many elements of engineering structures, in particular, those which carry moving loads (a track structure, a contact wire) are considered as a one-dimensional system (a string, a beam) interacting with a deformable foundation. To design structures resting on the ground, the following three foundation models are mainly used: 1) the Winkler model; 2) the elastic half-space model; 3 ) the combined model of an elastic foundation.

The most suitable model for practical purposes is the Winkler foundation, since the results of designing structures by using this model closely correspond to experimental data.

When studying the motion of loads along one-dimensional elastic systems, the foundation is assigned as linear-elastic (the Winkler foundation) or viscoelastic, thus enabling to make allowance for the possibility of its resistance to any force action. In this case the foundation is usually not considered as a dynamic system.

A.I. Vesnitsky [1] proposed a mathematical model generalizing the Winkler model with consideration for the inertial nature of an elastic foundation with which a string performing small transverse oscillations interacted.

The propagation of transverse waves in a string resting on an elastic-inertial foundation is described by a nonlinear system of two second-order differential equations as follows:

$$
\frac{\partial^{2} u_{1}}{\partial t^{2}}-c_{v}^{2}\left(1+\frac{3}{2}\left(\frac{\partial u_{1}}{\partial x}\right)^{2}\right) \frac{\partial^{2} u_{1}}{\partial x^{2}}+\frac{\gamma}{\rho_{0} S}\left(u_{1}-u_{2}\right)=0,
$$

\footnotetext{
*Corresponding author: aleonav@mail.ru
} 


$$
\frac{\partial^{2} u_{2}}{\partial t^{2}}+\frac{2 \gamma}{\rho_{g}} u_{2}=\frac{\gamma}{\rho_{g}} u_{1}
$$

where $u_{1}(x, t), u_{2}(x, t)$ are transverse deviations of the string and the center line of the foundation from the equilibrium position, $\rho_{0}, S$ are the cubic density and the crosssectional area of a one-dimensional flexible inextensible string, $\gamma, \rho_{g}$ are the stiffness and the linear mass of the elastic-inertial foundation, $c_{v}=\sqrt{N /\left(\rho_{0} S\right)}$ is the velocity of the transverse wave propagation in a string without an elastic-inertial foundation. At the initial instant the tension force of the string is equal to $N$ and the string position coincides with the $x$ axis.

The first equation of the system describes waves propagating along the string, the second one specifies the dynamics of the elastic-inertial foundation interacting with the string. The dispersion properties of the system under consideration were studied in paper $[2]$.

The system of equations (1), (2) is reduced to a single equation relative to transversal displacements of the string:

$$
\frac{\partial^{2} U}{\partial \tau^{2}}-a_{2} \frac{\partial^{2} U}{\partial z^{2}}+a_{3} \frac{\partial^{4} U}{\partial \tau^{4}}-a_{4} \frac{\partial^{4} U}{\partial \tau^{2} \partial z^{2}}+a_{5} U-a_{6}\left(\frac{\partial U}{\partial z}\right)^{2} \frac{\partial^{2} U}{\partial z^{2}}-a_{7} \frac{\partial^{2}}{\partial \tau^{2}}\left(\left(\frac{\partial U}{\partial z}\right)^{2} \frac{\partial^{2} U}{\partial z^{2}}\right)=0
$$

where $U=u_{1} / u_{0}$ is a dimensionless displacement, $z=x / X, \tau=t / T$ are nondimensional coordinate and time values, characteristic values are assumed to be equal to $T^{2}=\left(\varepsilon \rho_{g} / \gamma\right)\left(1+2 \rho_{0} S / \rho_{g}\right), X^{2}=(2 \varepsilon / \gamma) \rho_{0} S c_{v}^{2}, a_{i}(i=2 . .7)$ are dimensionless complexes

$$
\begin{aligned}
& a_{2}=1, a_{3}=\frac{\rho_{0} S}{\varepsilon \rho_{g}}\left(1+\frac{2 \rho_{0} S}{\rho_{g}}\right)^{-2}, a_{4}=\frac{1}{2 \varepsilon}\left(1+\frac{2 \rho_{0} S}{\rho_{g}}\right)^{-1}, \\
& a_{5}=\varepsilon, a_{6}=\frac{3 u_{0}^{2} \gamma}{4 \varepsilon \rho_{0} S c_{v}^{2}}, a_{7}=\frac{3 u_{0}^{2} \gamma}{8 \varepsilon^{2} \rho_{0} S c_{v}^{2}}\left(1+\frac{2 \rho_{0} S}{\rho_{g}}\right)^{-1} .
\end{aligned}
$$

While assuming further that the nonlinearity is small, we consider different cases of the ratios of the string mass $\rho_{0} S$ and the linear mass of the elastic-inertial foundation $\rho_{g}$.

If the linear mass of the string substantially exceeds the linear mass of the elasticinertial foundation $\left(\rho_{0} S>>\rho_{g}\right)$, we may assume that $1 /\left(1+2 \rho_{0} S / \rho_{g}\right) \sim \varepsilon^{2}$, in this case the nonlinearity is assumed to be a small quantity of the second order $3 u_{0}^{2} \gamma /\left(4 \rho_{0} S c_{v}^{2}\right) \sim \varepsilon^{2}$. Being limited to summands of the first-order of smallness, equation (3) takes the following

$$
\frac{\partial^{2} U}{\partial \tau^{2}}-a_{2} \frac{\partial^{2} U}{\partial z^{2}}-a_{4} \frac{\partial^{4} U}{\partial \tau^{2} \partial z^{2}}+a_{5} U-a_{6}\left(\frac{\partial U}{\partial z}\right)^{2} \frac{\partial^{2} U}{\partial z^{2}}=0
$$


If the linear masses of the string and the foundation are of the same order $\rho_{0} S / \rho_{g} \sim 1$ and the nonlinearity is of the first order of smallness $3 u_{0}^{2} \gamma /\left(4 \rho_{0} S c_{v}^{2}\right) \sim \varepsilon$, then equation (3) in the zeroth-order approximation for a small parameter will be as follows:

$$
a_{3} \frac{\partial^{4} U}{\partial \tau^{4}}-a_{4} \frac{\partial^{4} U}{\partial \tau^{2} \partial z^{2}}-a_{7} \frac{\partial^{2}}{\partial \tau^{2}}\left(\left(\frac{\partial U}{\partial z}\right)^{2} \frac{\partial^{2} U}{\partial z^{2}}\right)=0
$$

After the double integration over the non-dimensional time (the integration constants are assumed to be zero) equation (5) is transformed to the following wave equation containing a cubic nonlinearity

$$
\frac{\partial^{2} U}{\partial \tau^{2}}-b_{1}\left(1+b_{2}\left(\frac{\partial U}{\partial z}\right)^{2}\right) \frac{\partial^{2} U}{\partial z^{2}}=0
$$

where $b_{1}=a_{4} / a_{3}, b_{2}=a_{7} / a_{4}$.

If the string mass is substantially inferior to the mass of the elastic-inertial foundation ( $\rho_{0} S<<\rho_{g}$ ),we assume that $\rho_{0} S / \rho_{g} \sim \varepsilon^{2}, 3 u_{0}^{2} \gamma /\left(4 \rho_{0} S c_{v}^{2}\right) \sim \varepsilon^{2}$. In this case equation (3), including summands up to the first order of smallness, shall take the following form:

$$
\frac{\partial^{2} U}{\partial \tau^{2}}-a_{2} \frac{\partial^{2} U}{\partial z^{2}}-a_{4} \frac{\partial^{4} U}{\partial \tau^{2} \partial z^{2}}-a_{7} \frac{\partial^{2}}{\partial \tau^{2}}\left(\left(\frac{\partial U}{\partial z}\right)^{2} \frac{\partial^{2} U}{\partial z^{2}}\right)=0 .
$$

Let us turn in equation (4) to a moving coordinate system $\xi=z-\sqrt{a_{2}} \tau, \eta=\varepsilon \tau$. We shall obtain in the first approximation for $\varepsilon$ the following evolutionary equation relative to $W=\partial U / \partial \xi$ :

$$
\frac{\partial}{\partial \xi}\left(\frac{\partial W}{\partial \eta}+b_{1} W^{2} \frac{\partial W}{\partial \xi}+b_{2} \frac{\partial^{3} W}{\partial \xi^{3}}\right)-b_{3} W=0
$$

where coefficients of the equation are equal to $b_{1}=a_{6} /\left(2 \varepsilon \sqrt{a_{2}}\right), b_{2}=\sqrt{a_{2}} a_{4} /(2 \varepsilon)$, $b_{3}=a_{5} /\left(2 \varepsilon \sqrt{a_{2}}\right)$.

Note that this equation is very similar to the nonlinear evolutionary equation for internal waves in a rotating ocean

$$
\frac{\partial}{\partial \xi}\left(\frac{\partial U}{\partial \eta}+\frac{3 c_{0}}{2 h} U \frac{\partial U}{\partial \xi}+\frac{c_{0} \beta}{6} \frac{\partial^{3} U}{\partial \xi^{3}}\right)=\frac{\Omega^{2}}{2 c_{0}} U,
$$

where $U$ is the free liquid surface perturbation, $h$ is the depth, $c_{0}$ is the perturbation propagation velocity, $\beta$ is the high-frequency dispersion parameter, $\Omega$ is the Coriolis parameter characterizing the liquid rotation.

Equation (9) was first derived in 1978 by L.A. Ostrovsky [3] and is called the Ostrovsky equation. With $\Omega=0$ equation (9) is reduced to the Korteweg-de Vries equation (KdV) but 
as distinct from the latter it is not integrated by the method of the inverse scattering problem and does not have exact soliton solutions.

Equation (9) relates to a wide range of nonlinear systems characterized by the availability of a nondispersive band in the frequency spectrum, which separates regions with low- and high-frequency dispersion. Examples of such systems may be extraordinary electromagnetic and oblique magnetosonic waves in magnetized plasma; perturbations in an atom chain described by the Frenkel-Kontorova model; waves in transmission lines of the band-pass filter type.

Equation (8), as distinct from the Ostrovsky equation (9) contains not a quadratic but a cubic nonlinearity. It is seen that with $b_{3}=0$ equation (8) is reduced to the modified Korteweg-de Vries equation ( $\mathrm{mKdV}$ ), which, as is known, differs from the $\mathrm{KdV}$ equation by a higher degree of nonlinearity. Since the classical Ostrovsky equation under the derivative sign contains the $\mathrm{KdV}$ equation, equation (8) may by analogy with the $\mathrm{KdV}$ equations be called the modified Ostrovsky equation.

It is known that the Ostrovsky equation has no exact solutions [4] but assumes the qualitative investigation with the higher derivative to be equal to zero [5]. Equation (8) has no exact solutions either. Let us consider the modified Ostrovsky equation for $b_{2}=0$ :

$$
\frac{\partial}{\partial \xi}\left(\frac{\partial W}{\partial \eta}+b_{1} W^{2} \frac{\partial W}{\partial \xi}\right)-b_{3} W=0 .
$$

Let us further analyze the stationary solutions for equation (10) assuming $\chi=\xi-v \eta$ $(v>0)$. The equation of phase trajectories is as follows:

$$
\left(\frac{d W}{d \chi}\right)^{2}=\frac{b_{3}}{2 b_{1}}+\frac{C_{0}}{\left(b_{1} W^{2}-v\right)^{2}},
$$

where $C_{0}$ is the integration constant. Equation (11) has asymptotes: $W= \pm \sqrt{v / b_{1}}$, $d W / d \chi= \pm \sqrt{b_{3} /\left(2 b_{1}\right)}$. Phase portraits will be qualitatively different depending on values of the equation coefficients. For the mechanical system under study the coefficients shall be as follows $b_{1}=3 u_{0}^{2} \gamma /\left(8 \varepsilon^{2} \rho_{0} S c_{v}^{2}\right)>0, b_{3}=1 / 2>0$. In the system there are some limited movements (Fig. 1). Equation (8) has a solution in the form of a periodic stationary wave.

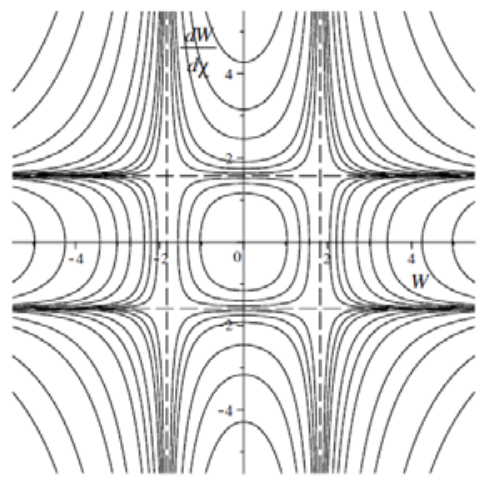

Fig. 1. Phase portraits $(W, d W / d \chi)\left(b_{1}>0\right.$, $\left.b_{3}>0\right)$

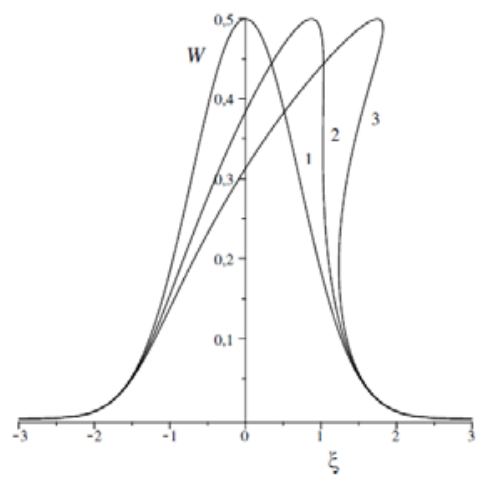

Fig. 2. Wave profiles $W\left(\xi, \eta_{1}\right)(1), W\left(\xi, \eta_{2}\right)$ (2), $W\left(\xi, \eta_{3}\right)(3)\left(\eta_{1}<\eta_{2}<\eta_{3}\right)$ 
Let us turn in equation (6) to the moving coordinate system $\xi=z-\sqrt{b_{1}} \tau, \eta=\varepsilon \tau$. We shall obtain in the first approximation for $\varepsilon$ the following evolutionary equation relative to $W=\partial U / \partial \xi$ :

$$
\frac{\partial W}{\partial \eta}+d W^{2} \frac{\partial W}{\partial \xi}=0
$$

where $d=\sqrt{b_{1}} b_{2} /(2 \varepsilon)=\left(3 u_{0}^{2} \gamma / 8 \varepsilon^{2} \rho_{0} S c_{v}^{2}\right) \sqrt{1+\rho_{g} /\left(2 \rho_{0} S\right)}$. Equation (12) may be classified as the Riemann equation with a cubic nonlinearity.

Equation (12) is included in quasilinear partial differential equations and may be solved by the method of characteristics. By replacing a variable, equation (12) shall be transformed to the following one

$$
\frac{\partial W}{\partial \eta}+W^{2} \frac{\partial W}{\partial \xi}=0 .
$$

If as the initial condition the Gaussian impulse is assigned $W(\xi, 0)=k_{1} \exp \left(-k_{2} \xi^{2}\right)$, the solution of equation (13) and the equation of characteristics shall acquire the following form:

$$
\begin{gathered}
W(\xi, \eta)=k_{1} \exp \left(-k_{2}\left(\xi-W^{2} \eta\right)^{2}\right), \\
\xi=\chi+k_{1}^{2} \exp ^{2}\left(-k_{2} \xi^{2}\right) \eta,
\end{gathered}
$$

where $\chi$ is the equation parameter with the change thereof we obtain the locus of characteristic lines on the plane $(\xi, \eta)$.

The wave profiles (14) at different instants of time are shown in Fig. 2. The wave profiles of the classical Riemann equation $V_{\eta}+V V_{\xi}=0$ (the simple wave equation) with the same initial condition are shown in Fig. 3. It may be seen in the Figures that the overturning occurs in the wave described by equation (13) and the same happens with the Riemann wave.

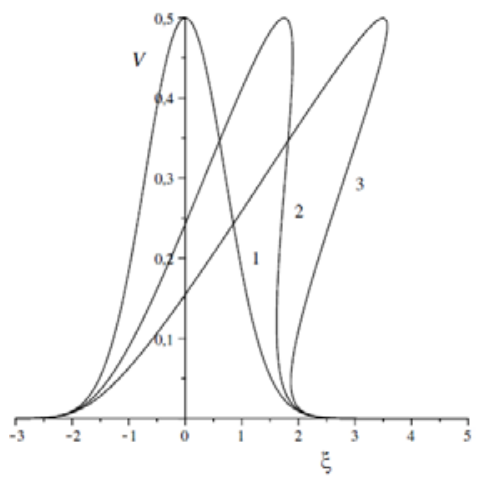

Fig. 3. The Riemann wave profiles at time instants $\eta_{1}(1), \eta_{2}(2), \eta_{3}$ (3)

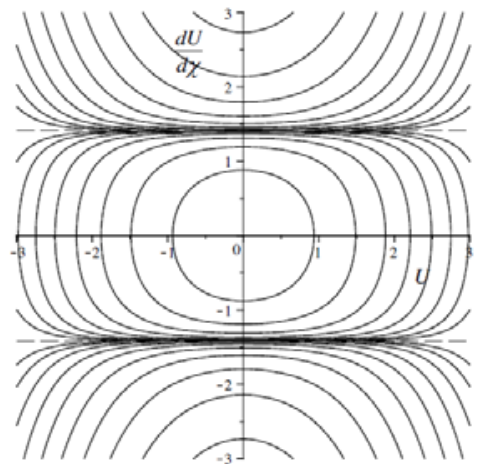

Fig. 4. Phase portrait of $(U, d U / d \chi)$ with $V<1$ 
A time instant when characteristic straight lines (15), intersect for the first time is equal to $t^{*}=\left(1 / 2 k_{1}^{2}\right) \sqrt{\exp (1) / k_{2}}$. The ratio of overturning time instants is equal to $t_{R}^{*} / t^{*}=\sqrt{2} k_{1}$, where $t_{R}^{*}$ is the time of the Riemann wave overturning. It follows therefrom that the time of the Riemann wave overturning is greater than the time of overturning for wave $W(\xi, \eta)$ with $k_{1}>\sqrt{2} / 2$, the time of the Riemann wave overturning is less with $k_{1}<\sqrt{2} / 2$ and the time instansts of overturning for waves are equal $\left(t_{R}^{*}=t^{*}\right)$ with $k_{1}=\sqrt{2} / 2$.

Thus, it is shown that the value of the initial perturbation amplitude affects the wave overturning time and propagation velocity. If the wave amplitude at the initial time instant is greater (less) than a certain critical value, the Riemann wave overturning occurs later (earlier) as compared to the wave described by the Riemann equation with a cubic nonlinearity.

Searching for the solution of equation (7) in the form of a traveling stationary wave $U=U(\chi)$, where $\chi=z-V \tau$, we pass to the fourth-order ordinary differential equation

$$
2 \varepsilon\left(1-V^{2}\right) \frac{d^{2} U}{d \chi^{2}}+V^{2} \frac{d^{2}}{d \chi^{2}}\left[\left(1+\frac{3 u_{0}^{2} \gamma}{4 \varepsilon \rho_{0} S c_{v}^{2}}\left(\frac{d U}{d \chi}\right)^{2}\right) \frac{d^{2} U}{d \chi^{2}}\right]=0 .
$$

This equation may be double integrated for $\chi$, if the integration constants are assumed to be equal to zero, then it is transformed as follows:

$$
\frac{d^{2} U}{d \chi^{2}}+d_{1} U-d_{2} U\left(\frac{d U}{d \chi}\right)^{2}=0
$$

where $d_{1}=2 \varepsilon\left(1-V^{2}\right) / V^{2}, d_{2}=d_{1} b, \quad b=3 u_{0}^{2} \gamma /\left(4 \varepsilon \rho_{0} S c_{v}^{2}\right)>0$, here $V$ is the nonlinear wave velocity. Equation (16) describes oscillations of an anharmonic oscillator with a cubic nonlinearity. The phase portrait for the case of $V<1$ is shown in Fig. 4. On the phase plane there are direct separating motions (asymptotes): $d U / d \chi= \pm \sqrt{d_{1} / d_{2}}$ (in the Figure they are marked with dotted lines).

It follows from the phase portrait analysis that in the subsonic case there are only nonlinear periodic (quasi-harmonic, cnoidal) waves and there are no solitary waves. In the supersonic case nonlinear waves do not propagate.

This work was supported by the Russian Science Foundation (project number 14-19-01637).

\section{References}

1. A.I. Vesnitsky, Waves in systems with moving boundaries and loads (Moscow, 2001) (in Russian)

2. V.I. Erofeev, D.A. Kolesov, E.E. Lisenkova, Acoust. Ph., 62(6), pp. 643-650 (2016)

3. L.A. Ostrovsky, Oceanology, 18, pp. 119-125 (1978)

4. M.L. Gandarias, M.S. Bruzón, Theoret. and Math. Phys., 168(1), pp. 898-911 (2011)

5. Y.A. Stepanyants, Chaos, Solitons and Fractals, 28, pp. 193-204 (2006) 